\title{
Association of the TREML2 and HTR1E Genetic Polymorphisms with Osteoporosis
}

\author{
Dongju Jung ${ }^{1}$ and Hyun-Seok Jin ${ }^{1,2} \uparrow$ \\ ${ }^{I}$ Department of Biomedical Laboratory Science, College of Life and Health Sciences, \\ Hoseo University, Asan, Chungnam 31499, Korea \\ ${ }^{2}$ The Research Institute for Basic Sciences, Hoseo University, Asan, Chungnam 31499, Korea
}

\begin{abstract}
Osteoporosis is one of the diseases caused by accumulation of effects from complex interactions between genetic and environmental factors. Aging is the major cause for osteoporosis, which normally increases skeletal fragility and bone fracture especially among the elder. "Omics" refers to a specialized research field dealing with high-throughput biological data, such as genomics, transcriptomics, proteomics or metabolomics. Integration of data from multi-omics has been approved to be a powerful strategy to colligate biological phenomenon with multiple aspects. Actually, integrative analyses of "omics" datasets were used to present pathogenesis of specific diseases or casual biomarkers including susceptible genes. In this study, we evaluated the proposed relationship of novel susceptible genes (TREML2, HTR1E, and GLO1) with osteoporosis, which genes were obtained using multi-omics integration analyses. To this end, SNPs of the susceptible genes in the Korean female cohort were analyzed. As a result, one SNP of HTR1E and five SNPs of TREML2 were identified to associate with osteoporosis. The highest significant SNP was rs6938076* of TREML2 (OR=0.63, CI: 0.45 0.89 , recessive $P=0.009$ ). Consequently, the susceptible genes identified through the multi-omics analyses were confirmed to have association with osteoporosis. Therefore, multi-omics analysis might be a powerful tool to find new genes associated with a disease. We further identified that TREML2 has more associated with osteoporosis in females than did HTRIE.
\end{abstract}

Key Words: Osteoporosis, High-throughput biological assays, TREML2, HTR1E, Genetic association study

\section{INTRODUCTION}

Osteoporosis is one of the diseases caused by accumulation of effects from complex interactions between genetic and environmental factors. Aging is the major cause for osteoporosis, which normally increases skeletal fragility and bone fracture especially among the elder. Osteoporosis is a common disease worldwide. Up to 49 million people were

\footnotetext{
*Received: August 31 30, 2015 / Revised: November 17, 2015

Accepted: November 30, 2015

${ }^{\dagger}$ Corresponding author: Hyun-Seok Jin. Department of Biomedical Laboratory Science, College of Life and Health Sciences, Hoseo University, Asan, Chungnam 31499, Korea.

Tel: +82-41-540-9968, Fax: +82-41-540-9997

e-mail: jinhs@hoseo.edu

C The Korean Society for Biomedical Laboratory Sciences. All rights reserved.
}

diagnosed osteoporosis in regions such as North America, Europe, Japan, and Australia (Wade et al., 2014). Osteoporosis occurs more frequently in females - prevalence of osteoporosis in females is about four times higher than that in males (Dhanwal et al., 2011; Wade et al., 2014). Similarly, $4.9 \%$ of males and $32.4 \%$ of females older than 50 years of age were diagnosed with osteoporosis according to the 2008 Korean National Health and Nutrition Examination Survey (Lee et al., 2013). Therefore, osteoporosis is a major public concern among the industrialized countries (Melton, 2003).

"Omics" refers to a specialized research field dealing with high-throughput biological data. Genomics, transcriptomics, proteomics and metabolomics are representative omics. Increase of accumulated omics data has become publicly 
and freely available; therefore it is possible to analyze biological phenomenon and diseases with multi-aspects by integrating the omics data. Furthermore, software methods for integrating each omics dataset are being developed (Zhang et al., 2010; Kirk et al., 2012; Xiong et al., 2012). Detection of known markers of a disease is a common process to validate a developed method.

A recently developed method to integrative omics datasets was reported. For identifying osteoporosis biomarkers, Lin et al. combined gene expression and SNP datasets with bone mineral density of 80 Caucasian females (GEO database accession number: GSE56815), 19 Caucasian females (GSE2208), 26 Chinese females (GSE7158), and 1,000 unrelated homogeneous Caucasians. Using the method, authors reported that TREML2, HTRIE and GLO1 were susceptible genes for osteoporosis (Lin et al., 2014). The three genes have not been reported to be associated with osteoporosis. Because the three genes are novel, we evaluated the developed method by analyzing association of SNPs of the genes with osteoporosis. We used SNPs of the three genes from Korean female cohort having osteoporosis. Using population-based statistical association analysis, we were able to verify TREML2 and HTR1E genes could be susceptible genes for osteoporosis in females as the omic data suggested.

\section{MATERIALS AND METHODS}

\section{Subjects}

The data from the subjects used in this study were collected from the Korean Association REsource (KARE) study, which was described in another report (Cho et al., 2009). Briefly, prospective community cohorts were recruited from two cities, Ansung and Ansan, Gyeonggi Province, Republic of Korea. Data of the community cohorts was obtained at 2001 as part of a major project for the Korean Health and Genome Study (KHGS) conducted and managed by Korea National Institute of Health (KNIH). Among the initial 10,038 subjects (40 69 years), 1196 subjects were excluded due to insufficiency for the criteria including sex inconsistency and individuals with tumors. Male subjects $(n=4,183)$ were excluded from the remaining 8,842 subjects because there were few cases of male osteoporosis in the community cohort. Among the remaining 4,659 female subjects, 855 subjects who were taking medicine were omitted because the bone density was likely to be changed by the medicine. Another 791 subjects who did not participate in the bone density measurement were excluded. Our study was carried out with the aged subjects $(50 \sim 69$ years, $n=$ $1,412)$ to focus on post-menopausal osteoporosis mainly caused by depletion of estrogen. The aged subjects were divided into normal controls $(\mathrm{n}=722)$ and osteoporosis case $(n=405)$. Subjects who had osteopenia were excluded. Basic characteristics of the subjects and their bone properties are described in Table 1. This study was approved by the institutional review board of the KNIH. Written informed consent was obtained from all of the subjects.

\section{Measurement of bone properties}

Speed of sound (SOS) value of bone was measured at distal radius and midshaft tibia using Omnisense 7000P quantitative ultrasound (QUS) device (Sunlight Medical Ltd, Tel-Aviv, Israel) to assess bone status (Weiss et al., 2000). Bone SOS value represents bone density and elasticity. The T-score was obtained by dividing SOS value between measured and mean of the healthy adult population by standard deviation (SD) of SOS value of an adult population. If the T-scores at distal radius (DR-SOS) or midshaft tibia (MTSOS) were less than $-2.6 \mathrm{SD}$ and $-3.0 \mathrm{SD}$, respectively, subjects were considered case subjects to distinguish them with control subjects according to the diagnostic categories established for adult women (Knapp et al., 2004). Subjects having T-scores of the DR-SOS and MT-SOS were higher than -1.4 SD and -1.6 SD, respectively, were considered control subjects following the criteria (Knapp et al., 2004).

\section{Genotyping and selection of SNPs}

Detailed information of genotyping, quality control processes and quantitative traits was described in the previous report (Cho et al., 2009). Briefly, DNA samples were isolated from peripheral blood of the participants and genotyped using the Affymetrix Genome-Wide Human SNP array 5.0 (Affymetrix, Inc., Santa Clara, CA, USA). Accuracy of the genotyping was calculated by Bayesian Robust Linear Modeling using the Mahalanobis Distance (BRLMM) algo- 
Table 1. Basic characteristics of the females in the KARE study cohort

\begin{tabular}{lccc}
\hline \multicolumn{1}{c}{ Characteristics } & \multicolumn{2}{c}{ Case-control analysis for osteoporosis } \\
\hline $\mathbf{2 0}$ year old females (age, $\mathbf{5 0 ~} \mathbf{6 9}$ years) & $\underline{\text { Controls }}$ & $\underline{\text { Cases }}$ & $P_{\text {-value }}{ }^{*}$ \\
No. & 722 & 405 & $<0.0001$ \\
Age (year) & $56.98 \pm 5.47$ & $61.84 \pm 4.70$ & 0.0013 \\
Body mass index (BMI) $\left(\mathrm{kg} / \mathrm{m}^{2}\right)$ & $24.54 \pm 2.93$ & $25.18 \pm 3.28$ & $<0.0001$ \\
Distal radius speed of sound (DR-SOS) (m/s) & $4224 \pm 146$ & $3972 \pm 185$ & $<0.0001$ \\
Midshaft tibia speed of sound (MT-SOS) (m/s) & $3920 \pm 105$ & $3600 \pm 115$ & $<0.0001$ \\
DR-SOS T-score & $0.41 \pm 1.21$ & $-1.72 \pm 1.57$ & $<0.0001$ \\
MT-SOS T-score & $-0.26 \pm 0.96$ & $-3.33 \pm 1.11$ & \\
\hline
\end{tabular}

*Significant differences in characteristics between the controls and cases were determined by the two-tailed Student's $t$-test.

rithm (Rabbee \& Speed, 2006). Samples that had genotyping accuracy were lower than $98 \%$, high missing genotype call rates ( $\geq 4 \%$ ), high heterozygosity ( $>30 \%$ ), or gender biases were excluded.

The 19 SNPs in GLO1, TREML2, and HTRIE genes that we analyzed were selected from the KARE data, based on their positions within the gene boundary $(5 \mathrm{~kb}$ upstream and downstream from the first and last exons, respectively) (Table 2). The positions of the SNPs were validated in the NCBI human genome build 36 . We also selected 16 imputed SNPs in TREML2 gene and 33 imputed SNPs in HTR1E by genotype imputation analysis using MACH 1.0.16 (Li et al., 2010) (Fig. 1). The Han Chinese from Beijing (CHB) and Japanese in Tokyo (JPT) individuals obtained from the Phase II HapMap database (release 24) (International HapMap Consortium, 2003) were used as references. The imputed SNPs with minor allele frequency $<0.01$ or coefficient of determination values $<0.5$ were excluded. The clinical information and genotype data that we used were graciously provided by the Center for Genome Science, KNIH, Korea Center for Disease Control (KCDC).

\section{Statistical analysis}

Statistical analyses were performed using PLINK version 1.07 (http://pngu.mgh.harvard.edu/ purcell/plink) and PASW Statistics version 18.0 (SPSS Inc., Chicago, IL, USA). Logistic regression analysis was used for the osteoporosis casecontrol association analysis in which age and residential area were included as covariates. The association analyses were based on additive, dominant, and recessive genetic model. $P$-values were not adjusted for multiple tests. Statistical significance was confirmed when its $P$-values with twotailed analysis were lower than 0.05 . For regional association plots, LocusZoom Version 1.1 (http://csg.sph.umich.edu/ locuszoom) were used, which is a web-based plotting tool using the CHBJPT (Chinese and Japanese) population panel originated from HapMap database.

\section{RESULTS}

\section{Basic subject characteristics}

The basic characteristics of the females from the KARE study cohort are shown in Table 1. Considering the mean age of menopause of Korean females, we selected female subjects who were more than 50 years old. A total of 1,412 female subjects (aged 50 69) belonged to this category. These females subjects were divided into two groups based on their SOS T-scores for osteoporosis case-control analysis: 722 normal controls and 405 osteoporosis cases. As expected, there were significant differences between the control and the case subjects in all of the basic characteristics we observed including mean age, body mass index (BMI), DRSOS, MT-SOS, DR-SOS T-score, and MT-SOS T-score, as determined by the two-tailed Student's $t$-test. Lower values of DR-SOS, MT-SOS, DR-SOS T-score, MT-SOS T-score and higher values of age and BMI were observed in the 


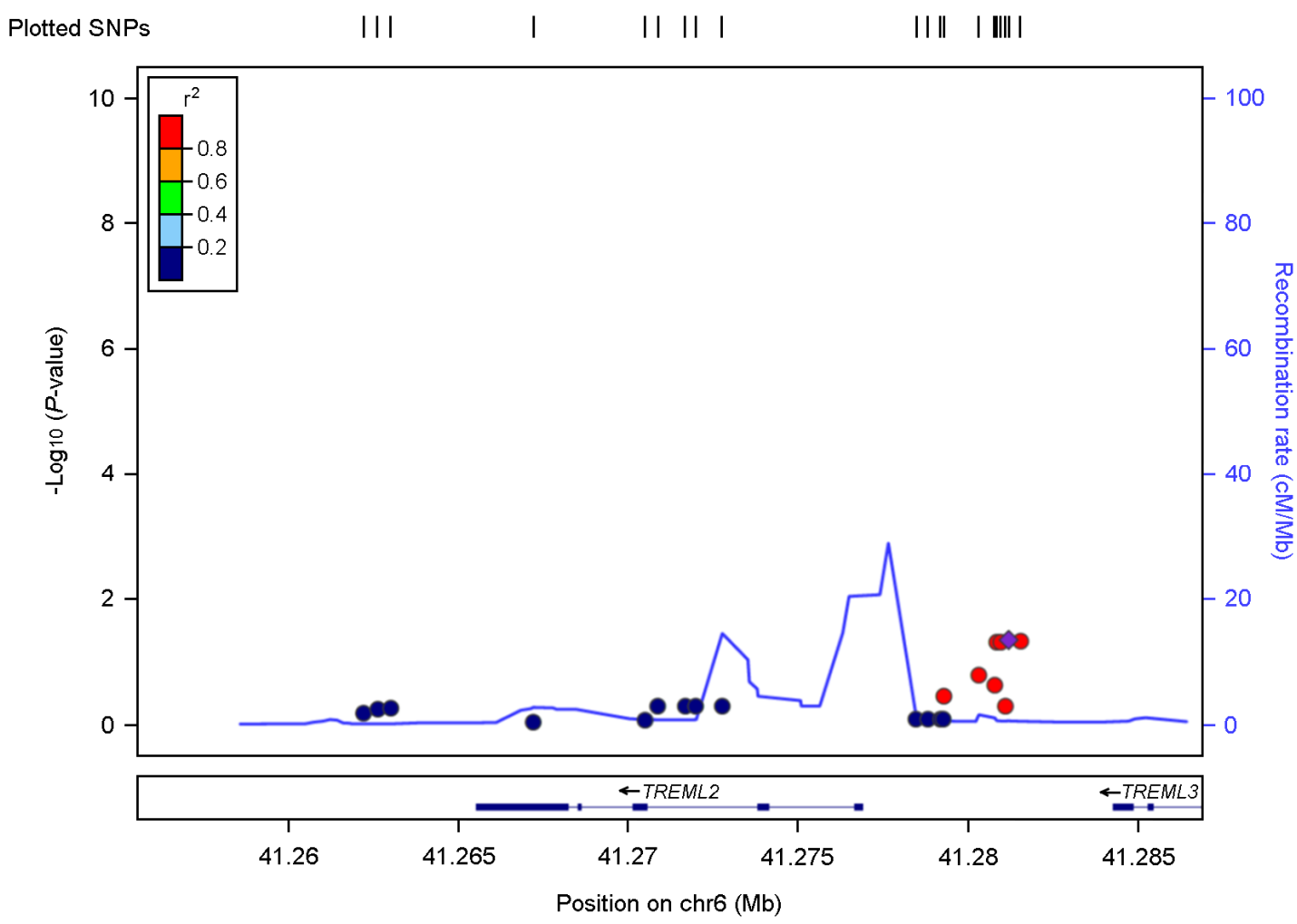

Fig. 1. Plots for the association result of the TREML2 SNPs with osteoporosis in the KARE female subjects. The position of the SNPS is shown at the top, and association results between SNPs in the TREML2 gene and osteoporosis in female subjects from the KARE study cohort are shown in the middle. The statistical significance ( $\log _{10} P$-value) of the analyzed $21 \mathrm{SNPs}$ is plotted, which are compose of experimentally genotyped 5 SNPs and in silico genotyped (imputed) 16 SNPs. The recombination rate estimated from the HapMap CHB (Han Chinese from Beijing) and JPT (Japanese from Tokyo) population data is shown by a blue line. The purple diamond with a SNP number (rs6938568) and $P$-value represents the SNPs most strongly associated with osteoporosis, and its correlated SNPs are shown in the indicated colors according to the levels of linkage disequilibrium $\left(r^{2}\right)$. At the bottom, the position $(\mathrm{Mb})$ of the TREML2 gene on chromosome 6 (NCBI build 36) is shown along with the gene structures.

osteoporosis females (Table 1).

\section{Association analysis with SNPs in GLO1, TREML2, and HTR1E genes with osteoporosis}

We selected and imputed SNPs of the novel susceptible genes from the KARE genotype data. We were able to find five, five, and nine SNPs of GLO1, TREML2, and HTR1E, respectively, by screening around their gene locus boundary (transcript $\pm 5 \mathrm{~kb}$ ) (Table 2). We first examined association of the SNPs with osteoporosis. SNP rs6938568 of TREML2 and SNP rs10944288 of HTRIE were found to have significant association with osteoporosis (Table 3). On the other hand, there were no SNPs of GLO1 having association with osteoporosis. Based on the results, we further selected 49 imputed SNPs (16 SNPs of TREML2, 33 SNPs of HTR1E) using MACH 1.0.16. Among the imputed SNPs of TREML2, three SNPs (rs9381043*, rs6938076*, and rs6939332*) were shown to have statistically significant association in the various genetic model, whereas there was no imputed SNPs of HTRIE showed association (Table 2, 3, and Fig. 1). More detailed information of the SNPs genes were listed in Table 2.

As a result, the one SNP of HTR1E and the five SNPs of TREML2 were associated with osteoporosis status. The SNP showed highest significant association with osteoporosis was rs6938076* of TREML2 (OR=0.63, CI: 0.45 0.89, recessive $P=0.009$ ) (Table 3 ). And other four SNPs (rs3957243, rs9381043*, rs6938568, and rs6939332*) of TREML2 were shown to have very similar values each other toward odd 
Table 2. Information on the GLO1, TREML2, and HTR1E gene' single nucleotide polymorphisms (SNPs) analyzed in this study

\begin{tabular}{|c|c|c|c|c|c|c|c|c|}
\hline $\begin{array}{l}\text { Gene symbol } \\
\text { (gene name, location) }\end{array}$ & No. & SNP & $\begin{array}{l}\text { Position, } \\
\text { bp }\end{array}$ & $\begin{array}{l}\text { Minor } \\
\text { allele }\end{array}$ & MAF & $\begin{array}{l}\text { Genotype } \\
\text { missing rate }\end{array}$ & HWE-p & $\begin{array}{l}\text { Functional } \\
\text { consequence }\end{array}$ \\
\hline \multirow{5}{*}{$\begin{array}{l}\text { GLO1 } \\
\text { (glyoxalase I, } \\
\text { 6p21.3-p21.1) }\end{array}$} & 1 & rs2736655 & 38756772 & A & 0.382 & 0.001 & 0.499 & Intron \\
\hline & 2 & rs4714175 & 38758819 & G & 0.331 & 0.007 & 0.228 & Intron \\
\hline & 3 & rs6932648 & 38766861 & A & 0.177 & 0.012 & 0.854 & Intron \\
\hline & 4 & rs1781716 & 38771422 & G & 0.236 & 0.000 & 0.409 & Intron \\
\hline & 5 & rs17622621 & 38777162 & A & 0.089 & 0.004 & 0.600 & Intron \\
\hline \multirow{8}{*}{$\begin{array}{l}\text { TREML2 } \\
\text { (triggering receptor } \\
\text { expressed on myeloid } \\
\text { cells-like } 2,6 \mathrm{p} 21.1 \text { ) }\end{array}$} & 1 & rs2093395 & 41263004 & $\mathrm{C}$ & 0.325 & 0.000 & 0.370 & Downstream \\
\hline & 2 & rs3747742 & 41270496 & $\mathrm{C}$ & 0.344 & 0.000 & 0.358 & Missense \\
\hline & 3 & rs4711658 & 41270869 & $\mathrm{~T}$ & 0.373 & 0.000 & 0.180 & Intron \\
\hline & 4 & rs3957243 & 41280845 & $\mathrm{~T}$ & 0.497 & 0.000 & 0.269 & Upstream \\
\hline & 5 & rs $9381043^{*}$ & 41280963 & $\mathrm{~T}$ & 0.497 & 0.000 & 0.260 & Upstream \\
\hline & 6 & rs6938076 & 41281106 & $\mathrm{C}$ & 0.491 & 0.054 & 0.032 & Upstream \\
\hline & 7 & rs6938568 & 41281198 & A & 0.496 & 0.012 & 0.014 & Upstream \\
\hline & 8 & rs6939332* & 41281553 & A & 0.497 & 0.000 & 0.251 & Upstream \\
\hline \multirow{9}{*}{$\begin{array}{l}\text { HTRIE } \\
\text { (5-hydroxytryptamine } \\
\text { (serotonin) receptor 1E, } \\
\text { G protein-coupled, } \\
\text { 6q14-q15) }\end{array}$} & 1 & rs1884740 & 87701171 & G & 0.070 & 0.000 & 0.253 & Upstream \\
\hline & 2 & rs 828360 & 87705285 & A & 0.148 & 0.000 & 0.801 & Intron \\
\hline & 3 & rs828358 & 87706172 & A & 0.297 & 0.001 & 0.879 & Intron \\
\hline & 4 & rs10944288 & 87708461 & $\mathrm{C}$ & 0.406 & 0.000 & 0.791 & Intron \\
\hline & 5 & rs16878041 & 87723212 & A & 0.336 & 0.000 & 0.703 & Intron \\
\hline & 6 & rs16878093 & 87736538 & $\mathrm{~T}$ & 0.335 & 0.000 & 0.417 & Intron \\
\hline & 7 & rs10485177 & 87759054 & G & 0.106 & 0.000 & 0.433 & Intron \\
\hline & 8 & rs16878128 & 87778426 & G & 0.342 & 0.002 & 0.813 & Intron \\
\hline & 9 & rs16878131 & 87780002 & A & 0.105 & 0.004 & 0.154 & Intron \\
\hline
\end{tabular}

*Imputed SNPs in the TREML2 gene. The SNPs were selected from the KARE data based on their locations within the gene boundary $(5 \mathrm{~kb}$ upstream and downstream of the first and last exons, respectively) according to NCBI human genome build 36. Abbreviations: HWE-p, frequency of dominant allele in Hardy-Weinberg equilibrium equation; MAF, minor allele frequency.

ratios and confidence intervals, because they commonly have homogeneity of minor allele frequency and reciprocally high correlation (Table 2, 3).

\section{DISCUSSION}

Combinatorial analyses of data obtained from omics are increasing becoming useful to identify susceptible genes for a disease. Using combinatorial analyses, three genes (TREML2, HTRIE, and GLOI) were identified as novel susceptible genes for osteoporosis. In this study, we examined whether the proposed multi-omics integration method was useful by analyzing the association of SNPs of the three genes with osteoporosis in a Korean female cohort. Based on the SNP relation, we were able to conclude that TREML2 and HTRIE had relation with osteoporosis in females (Table 3, Fig. 1). Lin et al. reported the three genes had relation with osteoporosis using multi-omics integration method by analyzing database worldwide (Lin et al., 2014). The absence of relation of GLO1 SNP with osteoporosis in our results suggests that SNP frequency in $G L O 1$ might represent ethnic difference or insufficient number of subjects having osteoporosis available in the KARE database.

The TREML2 gene locates on chromosome 6p21.1 encoding a 'Triggering Receptor Expressed on Myeloid cells-Like 2'. Although, TREM1 and TREM2 were known 
Table 3. Results of the case-control association analysis between the single nucleotide polymorphisms (SNPs) in the GLO1, TREML2, and HTRIE genes and osteoporosis in the KARE females

\begin{tabular}{|c|c|c|c|c|c|c|c|c|c|}
\hline \multirow{2}{*}{ Gene } & \multirow{2}{*}{ No. } & \multirow{2}{*}{ SNP } & \multirow{2}{*}{ A1 } & \multicolumn{2}{|c|}{ Additive model } & \multicolumn{2}{|c|}{ Dominant model } & \multicolumn{2}{|c|}{ Recessive model } \\
\hline & & & & OR $(95 \% \mathrm{CI})$ & $P$-value & OR $(95 \% \mathrm{CI})$ & $P$-value & OR $(95 \% \mathrm{CI})$ & $P$-value \\
\hline \multirow{5}{*}{ GLOI } & 1 & rs2736655 & $\mathrm{A}$ & $0.97(0.80 \sim 1.17)$ & 0.726 & $0.84(0.64 \sim 1.10)$ & 0.212 & $1.20(0.84 \sim 1.72)$ & 0.324 \\
\hline & 2 & rs4714175 & G & $0.99(0.81 \sim 1.21)$ & 0.922 & $0.91(0.69 \sim 1.19)$ & 0.478 & $1.21(0.80 \sim 1.84)$ & 0.364 \\
\hline & 3 & rs6932648 & A & $0.87(0.67 \sim 1.12)$ & 0.267 & $0.83(0.61 \sim 1.12)$ & 0.227 & $0.90(0.44 \sim 1.86)$ & 0.772 \\
\hline & 4 & rs1781716 & $\mathrm{G}$ & $1.01(0.82 \sim 1.25)$ & 0.921 & $1.02(0.78 \sim 1.33)$ & 0.899 & $1.00(0.59 \sim 1.68)$ & 0.997 \\
\hline & 5 & rs 17622621 & $\mathrm{~A}$ & $1.02(0.75 \sim 1.39)$ & 0.883 & $1.11(0.78 \sim 1.56)$ & 0.566 & $0.41(0.11 \sim 1.57)$ & 0.194 \\
\hline \multirow{8}{*}{ TREML2 } & 1 & rs2093395 & $\mathrm{C}$ & $1.00(0.82 \sim 1.23)$ & 0.979 & $1.09(0.83 \sim 1.42)$ & 0.553 & $0.81(0.52 \sim 1.27)$ & 0.362 \\
\hline & 2 & rs3747742 & $\mathrm{C}$ & $1.02(0.83 \sim 1.24)$ & 0.861 & $1.03(0.78 \sim 1.34)$ & 0.850 & $1.02(0.66 \sim 1.55)$ & 0.943 \\
\hline & 3 & rs4711658 & $\mathrm{T}$ & $1.01(0.83 \sim 1.23)$ & 0.937 & $1.10(0.83 \sim 1.44)$ & 0.507 & $0.85(0.56 \sim 1.27)$ & 0.417 \\
\hline & 4 & rs3957243 & $\mathrm{T}$ & $1.17(0.97 \sim 1.42)$ & 0.095 & $1.38(1.00 \sim 1.90)$ & 0.049 & $1.13(0.83 \sim 1.52)$ & 0.435 \\
\hline & 5 & rs9381043 ${ }^{*}$ & $\mathrm{~T}$ & $1.17(0.97 \sim 1.42)$ & 0.095 & $1.38(1.00 \sim 1.90)$ & 0.049 & $1.13(0.83 \sim 1.52)$ & 0.435 \\
\hline & 6 & rs6938076 & $\mathrm{C}$ & $0.82(0.68 \sim 1.00)$ & 0.049 & $0.90(0.66 \sim 1.23)$ & 0.508 & $0.63(0.45 \sim 0.89)$ & 0.009 \\
\hline & 7 & rs6938568 & A & $1.18(0.98 \sim 1.43)$ & 0.081 & $1.39(1.01 \sim 1.91)$ & 0.044 & $1.14(0.85 \sim 1.54)$ & 0.387 \\
\hline & 8 & rs6939332* & A & $1.17(0.97 \sim 1.42)$ & 0.096 & $1.39(1.01 \sim 1.91)$ & 0.046 & $1.12(0.83 \sim 1.52)$ & 0.452 \\
\hline \multirow{9}{*}{ HTRIE } & 1 & rs1884740 & $\mathrm{C}$ & $1.11(0.77 \sim 1.61)$ & 0.576 & $1.09(0.73 \sim 1.62)$ & 0.669 & $1.82(0.37 \sim 9.02)$ & 0.466 \\
\hline & 2 & rs 828360 & $\mathrm{~T}$ & $0.87(0.66 \sim 1.14)$ & 0.319 & $0.83(0.61 \sim 1.13)$ & 0.247 & $1.04(0.42 \sim 2.58)$ & 0.929 \\
\hline & 3 & rs828358 & A & $0.99(0.81 \sim 1.21)$ & 0.911 & $0.97(0.74 \sim 1.27)$ & 0.832 & $1.03(0.65 \sim 1.63)$ & 0.910 \\
\hline & 4 & rs 10944288 & $\mathrm{C}$ & $1.22(1.01 \sim 1.47)$ & 0.044 & $1.33(1.00 \sim 1.76)$ & 0.049 & $1.25(0.88 \sim 1.78)$ & 0.214 \\
\hline & 5 & rs16878041 & $\mathrm{T}$ & $0.97(0.79 \sim 1.18)$ & 0.738 & $0.97(0.74 \sim 1.27)$ & 0.838 & $0.92(0.59 \sim 1.42)$ & 0.696 \\
\hline & 6 & rs 16878093 & $\mathrm{~T}$ & $0.97(0.79 \sim 1.18)$ & 0.749 & $0.98(0.75 \sim 1.28)$ & 0.887 & $0.90(0.59 \sim 1.39)$ & 0.647 \\
\hline & 7 & rs 10485177 & G & $1.07(0.78 \sim 1.48)$ & 0.668 & $1.05(0.74 \sim 1.49)$ & 0.786 & $1.59(0.46 \sim 5.44)$ & 0.460 \\
\hline & 8 & rs 16878128 & G & $1.04(0.85 \sim 1.27)$ & 0.715 & $1.02(0.78 \sim 1.34)$ & 0.871 & $1.12(0.73 \sim 1.70)$ & 0.605 \\
\hline & 9 & rs 16878131 & A & $1.07(0.77 \sim 1.47)$ & 0.692 & $1.04(0.73 \sim 1.48)$ & 0.817 & $1.60(0.47 \sim 5.48)$ & 0.453 \\
\hline
\end{tabular}

*Imputed SNPs in the TREML2 gene. Age and residential area were included as covariates in all genetic models. $P$-values $<0.05$ are indicated in bold. Abbreviations: A1, minor allele; CI, confidence interval; MAF, minor allele frequency; OR, odds ratio.

to participate in the bone reabsorption metabolism (KlesneyTait et al., 2006; Otero et al., 2012), there had been no published article about TREML2 participation in osteoporosis until Lin et al. reported the relation (Lin et al., 2014). The HTRIE gene locates on chromosome 6q14-q15 encoding '5-Hydroxytryptamine Receptor 1E, G protein-coupled'. One SNP of HTRIE were reported to have relation with osteoporosis according to a GWAS for osteoporosis showing risk of nonvertebral fractures in females $(P=0.005)$ (Karasik et al., 2012). In our analyses, there was association between the SNP of HTRIE gene and osteoporosis in females, but only one $\mathrm{SNP}$ was statistically significant $(P=0.044)$. It might be reasonable to suggest that TREML2 is more related with osteoporosis in Korean females than HTRIE.
In addition to the multi-omics integration method, conventional genetic association studies, such as replication association study and candidate gene association study, have been used to find or evaluate susceptible genes for diseases (Jin et al., 2011; Jin \& Eom, 2012a; Jin et al., 2014; Jin \& Eom, 2012b). Our results suggest that the multi-omics integration method, which uses web sources of variations, gene expression, and various omics database, could be one of the conventional genetic association studies for identifying disease-related genes. In summary, the three genes (TREML2, $H T R I E$, and $G L O I$ ) that were reported to have relation with osteoporosis were evaluated their association with osteoporosis in Korean females via SNP-based genetic association method. Through the evaluation, we found i) the reliability 
of the multi-omics integration method for identifying noble genes associated with a disease, and ii) the association of TREML2 and HTR1E genetic polymorphisms with osteoporosis in Korean females.

\section{Acknowledgements}

This research was supported by Basic Science Research Program through the National Research Foundation of Korea (NRF) funded by the Ministry of Education (NRF2013R1A1A2010123).

\section{Conflict of interest}

The authors declare that they have no competing interests.

\section{REFERENCES}

Cho YS, Go MJ, Kim YJ, Heo JY, Oh JH, Ban HJ, Yoon D, Lee MH, Kim DJ, Park M, Cha SH, Kim JW, Han BG, Min H, Ahn Y, Park MS, Han HR, Jang HY, Cho EY, Lee JE, Cho NH, Shin C, Park T, Park JW, Lee JK, Cardon L, Clarke G, McCarthy MI, Lee JY, Lee JK, Oh B, Kim HL. A large-scale genome-wide association study of asian populations uncovers genetic factors influencing eight quantitative traits. Nat Genet. 2009. 41: 527-534.

Dhanwal DK, Dennison EM, Harvey NC, Cooper C. Epidemiology of hip fracture: Worldwide geographic variation. Indian J Orthop. 2011. 45: 15-22.

International HapMap Consortium. The international HapMap project. Nature. 2003. 426: 789-796.

Jin HS, Eom YB. Replicated association between SLC4A4 gene and blood pressure traits in the korean population. J Exp Biomed Sci. 2012a. 18: 377-383.

Jin HS, Eom YB. Genetic variations of ESR1 gene are associated with bone mineral density traits in korean women. J Exp Biomed Sci. 2012b. 18: 244-253.

Jin HS, Kim J, Lee SJ, Kim K, Go MJ, Lee JY, Lee HJ, Song J, Jeon BT, Roh GS, Kim SJ, Kim BY, Hong KW, Yoo YH, Oh $\mathrm{B}$, Kang Y, Jeong SY. The PARK2 gene is involved in the maintenance of pancreatic beta-cell functions related to insulin production and secretion. Mol Cell Endocrinol. 2014. 382: 178-189.

Jin HS, Hong KW, Kim BY, Kim J, Yoo YH, Oh B, Jeong SY. Replicated association between genetic variation in the PARK2 gene and blood pressure. Clin Chim Acta. 2011. 412: 1673
$-1677$.

Karasik D, Cheung CL, Zhou Y, Cupples LA, Kiel DP, Demissie S. Genome-wide association of an integrated osteoporosisrelated phenotype: Is there evidence for pleiotropic genes? J Bone Miner Res. 2012. 27: 319-330.

Kirk P, Griffin JE, Savage RS, Ghahramani Z, Wild DL. Bayesian correlated clustering to integrate multiple datasets. Bioinformatics. 2012. 28: 3290-3297.

Klesney-Tait J, Turnbull IR, Colonna M. The TREM receptor family and signal integration. Nat Immunol. 2006. 7: 1266-1273.

Knapp KM, Blake GM, Spector TD, Fogelman I. Can the WHO definition of osteoporosis be applied to multi-site axial transmission quantitative ultrasound? Osteoporos Int. 2004. 15: 367 -374 .

Lee YK, Yoon BH, Koo KH. Epidemiology of osteoporosis and osteoporotic fractures in South Korea. Endocrinol Metab (Seoul). 2013. 28: 90-93.

Li Y, Willer CJ, Ding J, Scheet P, Abecasis GR. MaCH: Using sequence and genotype data to estimate haplotypes and unobserved genotypes. Genet Epidemiol. 2010. 34: 816-834.

Lin D, Zhang J, Li J, He H, Deng HW, Wang YP. Integrative analysis of multiple diverse omics datasets by sparse group multitask regression. Front Cell Dev Biol. 2014. 2: 62.

Melton LJ, 3rd. Adverse outcomes of osteoporotic fractures in the general population. J Bone Miner Res. 2003. 18: 1139-1141.

Otero K, Shinohara M, Zhao H, Cella M, Gilfillan S, Colucci A, Faccio R, Ross FP, Teitelbaum SL, Takayanagi H, Colonna M. TREM2 and beta-catenin regulate bone homeostasis by controlling the rate of osteoclastogenesis. J Immunol. 2012. 188: 2612-2621.

Rabbee N, Speed TP. A genotype calling algorithm for affymetrix SNP arrays. Bioinformatics. 2006. 22: 7-12.

Wade SW, Strader C, Fitzpatrick LA, Anthony MS, O'Malley CD. Estimating prevalence of osteoporosis: Examples from industrialized countries. Arch Osteoporos. 2014. 9: 182.

Weiss M, Ben-Shlomo AB, Hagag P, Rapoport M. Reference database for bone speed of sound measurement by a novel quantitative multi-site ultrasound device. Osteoporos Int. 2000. 11: 688-696.

Xiong Q, Ancona N, Hauser ER, Mukherjee S, Furey TS. Integrating genetic and gene expression evidence into genomewide association analysis of gene sets. Genome Res. 2012. 22: 386-397.

Zhang K, Gray JW, Parvin B. Sparse multitask regression for identifying common mechanism of response to therapeutic targets. Bioinformatics. 2010. 26: i97-105. 